\title{
Virus Diseases*
}

$\mathrm{T}^{\mathrm{n}}$ HE term 'virus', formerly used in a much wider sense, has recently been given a special and restricted application. Viruses are distinguished from such long-recognised agents of infection as bacteria by their small dimensions, putting many of them beyond the limit of clear visibility by the highest powers of the microscope, and allowing them to pass through filters fine enough to retain the smallest ordinary bacteria; and by the fact that they cannot be cultivated on ordinary, non-living media. The work of Pasteur and Koch initiated an epoch of investigation on the relation of visible bacteria to different infections, so that the first clear recognition of the existence of ultramicroscopic and filterable infective agents came only in the last decade of the nineteenth century, and the present century has seen the rapid progress of investigations on their nature. The viruses first recognised - those of mosaic tobacco disease and the foot-and-mouth disease-are still far beyond the range of the most advanced microscopical methods, and, if studied by themselves, might still be regarded as living contagious principles in solution. A large number of diseases of man, animals and plants are now known to be due to infection by viruses, and methods now available have shown that the infective units of the different recognised viruses cover a wide range of dimensions.

Ordinary bacterial filters only indicate that the sizes of the infective units of viruses are below a certain maximum. Ordinary microscopical methods may show that the particles of a particular virus are beyond the range of resolution, or that it contains particles which can be brought just into the range of visibility (about $0 \cdot 2 \mu$ ) when loaded with a dye. Neither of these methods, however, enables the natural sizes of the units of different viruses to be determined for comparison.

Conventional microscopical methods revealed the association between infection with certain viruses and the appearance in the infected cells of the relatively large structures termed 'inclusion bodies'. These could not represent the virus in the form in which it passes a bacteriological filter. In 1904, however, Borrel described extremely minute bodies in suitably stained films from the juice of tissues infected with fowl-pox, and similar 'elementary corpuscles' were later found in material from vaccinia (by Paschen) and from Molluscum contagiosum. The important discovery was made in recent years (Goodpasture) that the large 'inclusion bodies' consist of 'elementary corpuscles' closely packed in a structureless matrix, and that a single washed

* Substance of a lecture entitied "Ultramicroscopic Organisms and the 'I'roubles which they cause", delivered by Sir Henry Dale, Sec. R.S., at Bedford College, London, on March 1 . inclusion body will transmit a virus infection. The evidence for identification of the elementary corpuscles as the organisms of these viruses has been strengthened by their agglutination with sera from animals immune to the corresponding virus (Leding. ham).

In the absence of any possibility of testing their identity with the virus by artificial culture, there remained that of comparing the sizes of fresh, unstained elementary corpuscles from different viruses, as measured on photographs of critical microscopic images, obtained with ultra-violet rays of a suitable wave-length (Barnard), with the sizes of the infective units of the corresponding viruses, as measured by differential filtration through graded collodion membranes, of which the effective pore dimensions could be determined with sufficient accuracy (Elford). The comparison of the measure. ments of visible corpuscles, on one hand, and infective units on the other, has now been completed in the case of several viruses, and has shown a correspondence so satisfactory as to leave no room for doubt that the elementary corpuscles are the organisms of these viruses.

Filtration measurements of the infective units of other viruses, however, show that they are much too small to be brought within the range of any microscopical method which can be foreseen. In the case of the virus of foot-and-mouth disease, the units appear to have a diameter as small as about $10 \mathrm{~m} \mu$, only about twice that of the molecule of oxyhæmoglobin. The difficulty of regarding such minute particles as self-propagating organisms has given rise to the suggestion that they are toxic principles, reproduced by the perverted metabolism of cells which they infect. A similar suggestion has been made to account for the reproduction of the virus-like lysins or bacteriophages which infect bacteria. In both cases, however, such a theory seems to be incompatible with recent evidence dealing with specific immunity to such viruses.

Specific treatment of viruses is still limited to the production or re-inforcement of a naturally acquired immunity. Recent success in that direction with dog-distemper (Laidlaw and Dunkin) has aroused in some minds a hope of similar success with a human disease, such as influenza. The analogy fails, unfortun. ately, through lack of an animal species in which the influenzavirus can be propagated and made the subject of deliberate experiment, and of evidence of a strong and lasting immunity to its attack. The facts, however, that methods for the exact study of viruses are only now becoming available, and that knowledge of their properties is of recent and rapid growth, justifies a general hope of much wider success in dealing with the diseases which they produce.

\section{Geology in Great Britain}

\section{$\mathrm{T}$} "HE "Summary of Progress" of the Geological 1 Survey of Great Britain for 1930 has been issued in the three parts that have now become customary, the first ${ }^{1}$ being devoted to the annual reports of the Geological Survey Board and of the Director and to the routine work carried out during the year under review, while the others contain papers on subjects of special interest. Forty-four maps were published with seven English and three Scottish memoirs, some of which have already been noticed in our columns (NATURE, vol. 127, 574, April 11, 1931).

of the Scottish memoirs, that dealing with Ardnamurchan deserves special mention. It is a work of unrivalled geological interest that will always rank as one of the greatest contributions to the 